\title{
Noninformative Vision Causes Adaptive Changes in Tactile Sensitivity
}

\author{
Justin A. Harris, Ehsan Arabzadeh, Clinton A. Moore, and Colin W. G. Clifford \\ School of Psychology, The University of Sydney, Sydney 2006, Australia
}

The last few years have witnessed a rapid growth of research investigating how information is integrated across sensory modalities, a process at the core of our everyday perceptual experiences. The present study focuses on the integration of vision and touch and, in particular, how tactile perception is affected by a view of the relevant body part containing no information about the tactile stimulus itself. Previous studies have established that this "noninformative vision" can improve subsequent tactile discrimination (Kennett et al., 2001; Taylor-Clarke et al., 2004a), a finding we confirm in the present study. However, we also report here that noninformative vision impairs the detection of tactile stimuli and the discrimination of near-threshold stimuli. These effects are shown to resemble, and indeed combine additively with, shifts in discrimination and detection thresholds produced by adaptation to suprathreshold tactile stimulation. We conclude that noninformative vision of the body does not simply enhance somatosensory processing, but rather it induces adaptive changes in tactile sensitivity via shifts in gain control operating within a bimodal sensory system. This constitutes a novel means by which vision of the body can alter tactile perception.

Key words: multisensory; touch; visuo-tactile; adaptation; discrimination; detection

\section{Introduction}

Investigations of visuo-tactile integration have examined how information from vision and touch are combined in perceptual experience (Lederman and Klatzky, 2004). These investigations have shown that people not only use both sources of information but, at least under certain circumstances, they combine the information optimally taking into account differences in the fidelity of each source (Ernst and Banks, 2002). Such studies are primarily concerned with how relevant information about a stimulus is combined from different modalities. However, recent evidence indicates that even irrelevant (noninformative) visual input can affect tactile perception. Haggard and colleagues (Kennett et al., 2001; Taylor-Clarke et al., 2004a) have published a series of papers in which they report that tactile acuity is enhanced if participants view the appropriate part of their body before or during the tactile stimulation. An important feature of these studies is that the visual input carried no information about the identity of the tactile stimulus and therefore the observed improvement in tactile sensitivity was not attributable to any direct contribution that vision could make to perception of the target stimulus. These researchers have argued that noninformative vision improves tactile perception by augmenting the somatosensory response to tactile stimulation (Taylor-Clarke et al., 2002; Haggard et al., 2003). The effect of noninformative vision on touch is important because it reveals a distinct type of cross-modal interaction that

Received Feb. 4, 2007; revised May 31, 2007; accepted May 31, 2007.

This work was supported by Australian Research Council Grants DP0343552 and DP0663086 and by a Human Frontiers Science Program Fellowship to E.A.

Correspondence should be addressed to Dr. Justin A. Harris, School of Psychology, The University of Sydney, Sydney 2006, Australia. E-mail: justinh@psych.usyd.edu.au.

DOI:10.1523/JNEUROSCI.2102-07.2007

Copyright $\odot 2007$ Society for Neuroscience $\quad$ 0270-6474/07/277136-05\$15.00/0 goes beyond previous evidence that information about a stimulus received in one modality can bias or assist perceptual decisions made in another modality.

The present study investigated how simple tactile perception is affected by noninformative vision of the relevant body part (in this case, the right hand). In previous studies, noninformative vision improved tactile acuity (measured as two-point discrimination thresholds or performance on a grating discrimination). This interaction may be specific to judgments about the spatial metrics of tactile stimuli, as suggested by recent evidence that the perceived distance between tactile stimuli can be altered when participants view distorted images of a body part (Taylor-Clarke et al., 2004b). If so, noninformative vision would not be expected to affect detection of a simple tactile stimulus, such as a vibration, or perception of its intensity, as related to the amplitude of the vibration. However, if noninformative vision leads to a general facilitation of somatosensory processing, then its effects on touch may extend beyond perception of spatial attributes to include detection and amplitude discrimination. This possibility is examined in the present experiments.

\section{Materials and Methods}

Experiments 1-4. Fifty-eight first-year psychology students at The University of Sydney participated for course credit $(n=20$ for experiments 1 and $2 ; n=9$ for experiments 3 and 4 ). They sat with their right hand facing palm up inside the test apparatus (Fig. 1). Square-wave vibrations were presented to the right index finger via a 3-mm-diameter steel rod driven by a small vibration excitor and power amplifier (type 4810 "minishaker" and type 2718 amplifier; Brüel \& Kjær, Nærum, Denmark) controlled by a computer. The finger was supported by a piece of molded rubber foam, and the flat tip of the vibrating rod produced a static indentation $(\sim 1 \mathrm{~mm})$ into the finger pad.

In experiments 1 and 3, detection thresholds were measured for each 

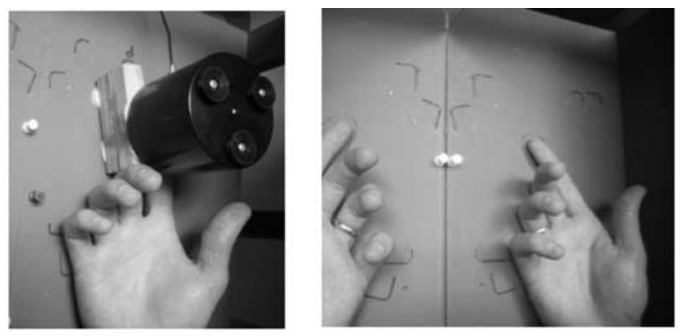

\section{2-AFC detection task}

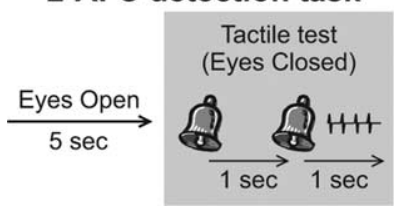

2-AFC discrimination task

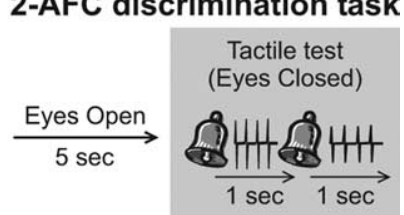

Figure 1. Top, Photographs showing the experimental setup. The participant's right hand was placed inside a box with the index finger extended to be in contact with the vibration device (left). During experiments $1-4$, the right hand and device were concealed from view by a mirror; the mirror either reflected the image of the participant's left hand to appear as the right hand (as shown on the right) or was turned around to create an opaque surface (not shown). In experiment 5, the participants either had direct view of their right hand (and stimulator) or these were concealed by a curtain. Bottom, Diagrams depicting trial structure for the twoalternative forced-choice (2-AFC) detection and discrimination tasks. On each trial, the participants reported which of two temporal intervals, signaled by two auditory cues, contained a vibration (detection task), or which interval contained the stronger of two vibrations (discrimination task).

participant using a three-down, one-up adaptive staircase procedure within a two-interval forced-choice task. On each trial, the participant was presented with a single $20 \mathrm{~Hz}$ vibration randomly occurring in one of two sequential intervals (marked by two auditory cues). The participant was required to say which interval contained the vibration. On the first trial of a staircase, the amplitude of the vibration was $16 \mu \mathrm{m}$. This was chosen to be suprathreshold for all participants. Whenever the participant identified the correct interval on three consecutive trials, the amplitude of the vibration was reduced; whenever the participant named the wrong interval, the amplitude was immediately increased. The amplitude was initially decreased in steps of $10 \%$ until the participant gave an incorrect response (reversal 1), at which point it was increased by steps of $5 \%$ until the next reversal (when the participant named three consecutive trials correctly), after which it was changed by $2 \%$, then $1 \%$, and finally in steps of $0.5 \%$ for the remainder of the staircase. The staircase was run until the participant completed 10 reversals, and the threshold for that staircase was taken as the average of the last 6 reversals.

To measure amplitude discrimination thresholds in experiments 2 and 4, the participant was presented on each trial with two $20 \mathrm{~Hz}$ vibrations in consecutive intervals (signaled by two auditory cues). The amplitude of the base vibration was $20 \mu \mathrm{m}$; the amplitude of the comparison vibration was always larger. The order in which they occurred was random on each trial. The participant's task was to say which vibration (first or second) had the larger amplitude. On the first trial of the staircase, the amplitude of the comparison vibration was $16 \mu \mathrm{m}$ above the base. This difference was progressively reduced according to the same algorithm described above for the measurement of detection thresholds.

In experiments 3 and 4 , a $20 \mathrm{~Hz}$ adapting vibration, $20 \mu \mathrm{m}$ in amplitude, was presented for $60 \mathrm{~s}$ at the beginning of each staircase. During the staircases, the adapting vibration was presented for $5 \mathrm{~s}$ between each trial to "top up" the level of adaptation. At the beginning and end of these experiments, unadapted baseline thresholds were obtained for each participant.

In all experiments, the participant's right hand and vibrotactile stimulator were located in the right half of a wooden box ( $46 \mathrm{~cm}$ wide, $22 \mathrm{~cm}$ tall, $38 \mathrm{~cm}$ deep), and the left hand was placed in the left side of the same box. The hand and stimulator were concealed from view by a wooden lid over the right half of the box and an angled mirror separating the left and right halves of the box (Fig. 1). The participant could see into the left side of the box. In the visible-hand condition, the mirror faced to the left, thus reflecting the left half of the interior of the box, including the participant's left hand. This created an apparent view of the right hand. The no-view condition was created by turning the mirror around so that the opaque surface faced out to the left, blocking any view of the right hand. The outline of a left hand (palm up) was drawn on the floor of the left side of the box, and the participants were instructed to place their left hand in that location, with the palm facing upward and fingers outstretched. In this position, the mirror reflection of the left hand was in the same position and posture as the right hand. During the visible-hand condition, participants were instructed to maintain fixation on the center of the index finger pad of the reflected hand. During the no-view condition, participants fixated on a mark in the center of the back of the mirror (approximately aligned with the position of the right index finger). Between each test trial, the participants looked at their finger (or the back of the mirror) for $5 \mathrm{~s}$ until the first auditory cue signaled them to close their eyes. Their eyes were thus closed for the duration of the test period; they reopened their eyes when given their response.

Experiment 5. Experiment 5 was designed as a within-subject replication of experiments 1-4. Five new subjects [two authors (J.A.H. and E.A.) and three naive participants] were tested extensively, such that they performed under all eight conditions defined by the $2 \times 2 \times 2$ factorial design (visible hand/no view $\times$ adapted/unadapted $\times$ high/low base amplitude). This allowed us to confirm the findings observed in experiments 1-4 and to directly test for possible interactions between the factors. The procedure was similar to that described above, except for the following details. Instead of manipulating vision using a mirror, subjects either had direct view of their tested finger and hand, or their hand was concealed behind a black curtain. This allowed us to establish that the effects observed in experiments 1-4 were not a peculiar consequence of viewing a reflected image of their opposite hand. Each subject's detection threshold was measured at the beginning of testing (as the average of four staircase tests) under no-view conditions, and these were used to better equate stimuli across subjects. Thus, all subsequent testing required the subjects to discriminate between the amplitudes of two vibrations: both vibrations either had low base amplitude ( 1 time the detection threshold) or high base amplitude (10 times the detection threshold). All thresholds were measured using a Bayesian adaptive staircase procedure (Kontsevich and Tyler, 1999), which provides an optimal estimate of threshold within 30 trials. This allowed us to run between two and four staircases on each subject under each of the eight experimental conditions. Finally, subjects were tested with $40 \mathrm{~Hz}$ sinusoidal vibrations that can be more accurately controlled by the vibration device.

\section{Results}

\section{Experiments 1-4}

Across participants, detection thresholds for the square-wave vibrations ranged between 0.5 and $2 \mu \mathrm{m}$. This is at the lower end of the range of values reported in previous studies (Talbot et al., 1968; LaMotte and Mountcastle, 1975; Tommerdahl et al., 2005; Mahns et al., 2006). Because our participants differed substantially in their baseline thresholds, we normalized their thresholds across the two conditions. To do this, for experiments 1 and 2 we divided each participant's threshold for each condition by the average for that participant across both conditions; for experiments 3 and 4, we divided thresholds for each condition by the nonadapted baseline threshold.

The top panels of Figure 2 show normalized thresholds from experiments 1 and 2. Viewing an image of the hand before the tactile stimuli (i.e., in the visible-hand condition) elevated detection thresholds but lowered discrimination thresholds compared with the no-view condition. Two-tailed paired $t$ tests confirmed these differences, both for detection thresholds $\left(t_{(19)}=2.16 ; p=\right.$ $0.044)$ and discrimination thresholds $\left(t_{(19)}=2.36 ; p=0.030\right)$.

In experiments 3 and 4 , detection and discrimination thresh- 
No view

Visible hand
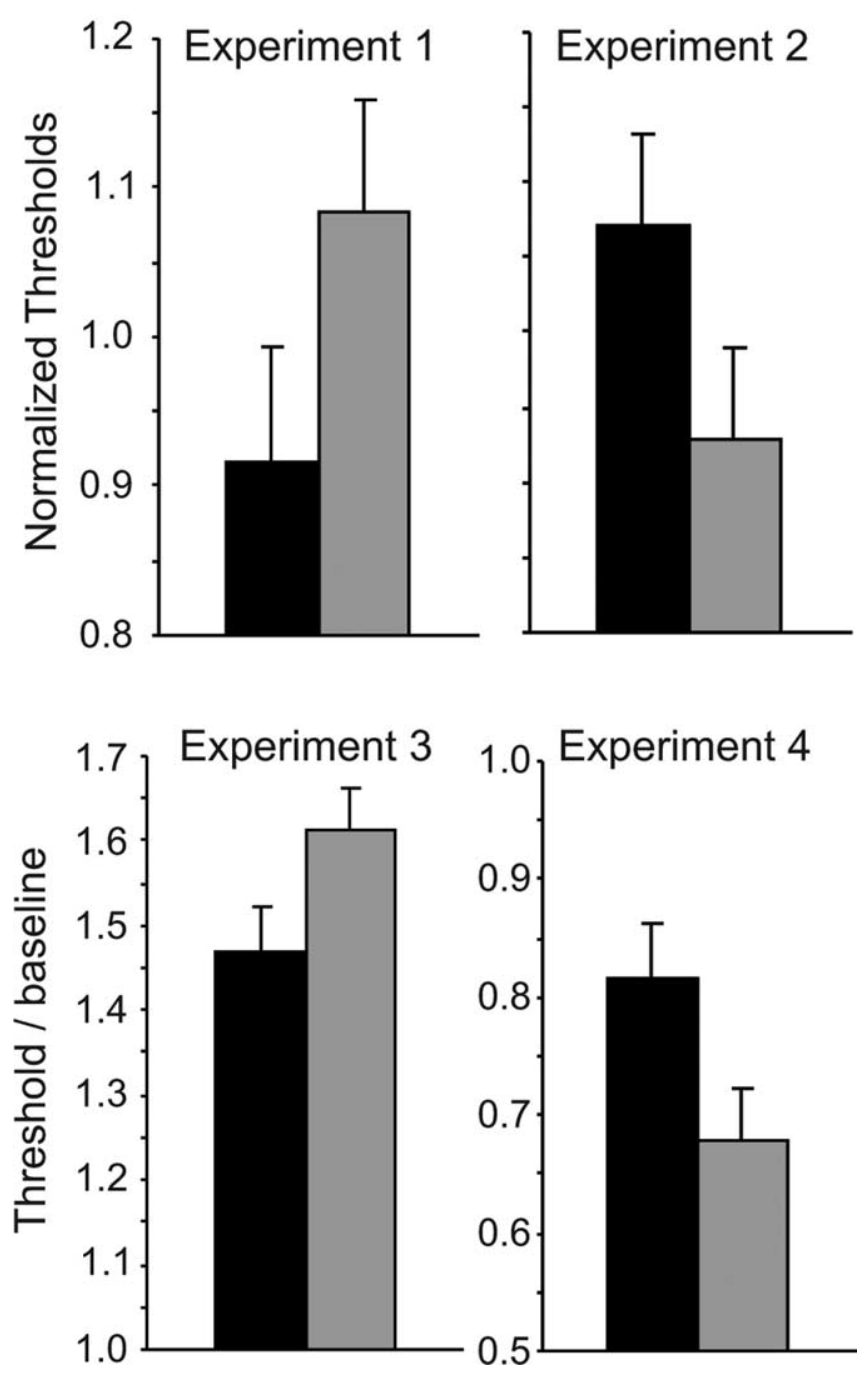

Figure 2. Top, Normalized thresholds for detection in experiment 1 (left) and for discrimination in experiment 2 (right). Bottom, Postadaptation detection thresholds in experiment 3 (left) and discrimination thresholds in experiment 4 (right) as a proportion of preadaptation baseline thresholds. Vertical bars show within-subject SEM $[\sqrt{ }(2 \times \mathrm{MSE} / n)]$ derived from paired $t$ tests comparing visible-hand and no-view conditions (Loftus and Masson, 1994). MSE, Mean square error.

olds were measured after a suprathreshold adapting vibration was presented to the tested fingertip. The adapting vibration elevated detection thresholds by $\sim 50 \%\left(t_{(8)}=4.23 ; p<0.001\right.$, comparing thresholds in the no-view condition against baseline thresholds measured without an adapting vibration), consistent with previous reports (Gescheider et al., 2001). The adapting vibration also lowered discrimination thresholds by $\sim 25 \%$ $\left(t_{(8)}=2.47 ; p=0.039\right)$, an effect that has been reported for discrimination of vibration frequency (Tommerdahl et al., 2005) but not, to our knowledge, for amplitude. These results are evident in the bottom panels of Figure 2, which show the detection and discrimination thresholds, as a proportion of unadapted baseline thresholds, for the visible-hand and no-view conditions. As in experiments 1 and 2, a view of the hand further elevated

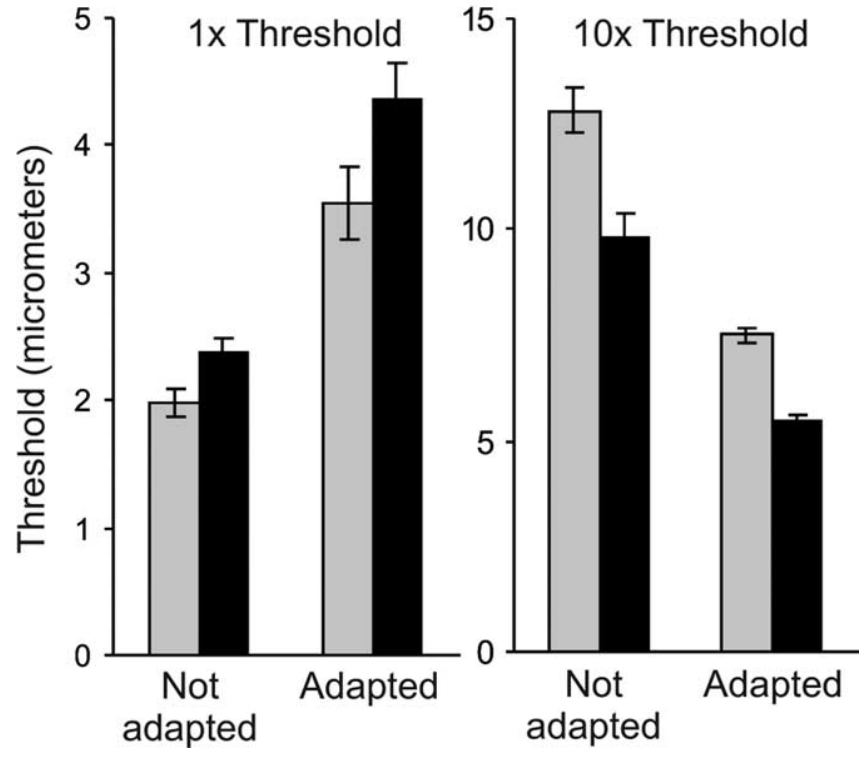

Figure 3. Discrimination thresholds in experiment 5. Subjects discriminated between lowamplitude vibrations (base amplitude $=1 \times$ detection threshold) and between higheramplitude vibrations (base amplitude $=10 \times$ detection threshold). Vertical bars show withinsubject SEM (see Fig. 2 for description).

detection thresholds $\left(t_{(8)}=2.72 ; p=0.026\right)$ but lowered discrimination thresholds $\left(t_{(8)}=3.00 ; p=0.017\right)$.

\section{Experiment 5}

Detection thresholds for the sinusoidal vibrations were higher than those obtained using square-wave vibrations (experiments 1-4) and ranged between 3.9 and $7.8 \mu \mathrm{m}$ (mean, 4.9). Mean discrimination thresholds during subsequent testing are shown in Figure 3. These are consistent with the findings from experiments 1-4. A $2 \times 2 \times 2$ ANOVA confirmed the following observations. Discrimination thresholds were significantly affected by base amplitude, being higher when the base was 10 times the detection threshold than 1 time detection threshold $\left(F_{(1,4)}=\right.$ $105.25 ; p<0.01)$. The effect of base amplitude interacted significantly with view $\left(F_{(1,4)}=92.54 ; p<0.01\right)$ and adaptation $\left(F_{(1,4)}=18.98 ; p=0.01\right)$, and there was no three-way interaction between these factors $(F<1)$, confirming that base amplitude had equivalent effects in modulating the impact of view and adaptation on discrimination thresholds. These effects were explored by two $2 \times 2$ ANOVAs conducted separately on data from high base amplitude and low base amplitude tests. These analyses confirmed that, when subjects were tested with low-amplitude vibrations, both view of the hand $\left(F_{(1,4)}=23.96 ; p<0.01\right)$ and adaptation $\left(F_{(1,4)}=48.67 ; p<0.01\right)$ increased discrimination thresholds, and these effects did not interact $\left(F_{(1,4)}=1.52\right.$; $p=0.29)$. Conversely, when subjects were tested with highamplitude vibrations, both view $\left(F_{(1,4)}=101.89 ; p<0.01\right)$ and adaptation $\left(F_{(1,4)}=11.65 ; p=0.03\right)$ reduced discrimination thresholds, and these effects did not interact $\left(F_{(1,4)}=2.38\right.$; $p=0.20)$. Finally, two-tailed paired $t$ tests confirmed that there was a significant difference between visible-hand and no-view conditions for each of the four testing combinations of base amplitude and adaptation (all $p<0.05$ ) 


\section{Discussion}

We measured people's thresholds to detect a vibration presented to their right index finger, or their thresholds to discriminate between the amplitudes of two sequential vibrations. The participants were tested with the tactile stimuli immediately after they viewed a mirror reflection of their left hand (producing the phenomenological experience of looking at the right hand) or immediately after they directly viewed their right hand. Their performance under these conditions was compared with their performance immediately after they viewed an opaque surface (the back of a mirror or black curtain) placed in front of their right hand. Thus, the first condition ("visible hand") provided a noninformative view of the tested body part (the participant viewed the stimulated fingertip but not the stimulus); the second condition ("no view") served as a control measure of tactile sensitivity. In addition, we investigated how the effect of these visual manipulations interacted with tactile adaptation induced by a suprathreshold vibration presented before the test stimulus.

Compared with the no-view condition, viewing the hand immediately before presentation of the test vibrations increased thresholds for detection and for discrimination between vibrations at detection threshold but decreased thresholds for discriminating between higher-amplitude vibrations ( 10 times the detection threshold). The same changes in threshold were induced by presentation of a suprathreshold vibration immediately before the test stimulus. Indeed, the effects produced by viewing the hand or adapting to a suprathreshold vibration were additive: viewing the hand changed thresholds over and above the equivalent changes induced by adaptation to the suprathreshold vibration, and the two factors did not interact (i.e., vision produced the same magnitude of effect with or without adaptation).

The above results appear, at first, contradictory, in that a noninformative view of the hand improved the ability of subjects to discriminate between two high-amplitude vibrations, but it impaired their ability to detect a low-amplitude vibration or discriminate between two near-threshold vibrations. The improvement in discrimination replicates the effect reported by Kennett et al. (2001), but the impairment in detection and near-threshold discrimination indicates that the effect of noninformative vision cannot be attributed to a general facilitation of tactile processing [a detrimental effect of vision on tactile detection has also been reported by Press et al. (2004)]. This particular pattern of results does, however, lend itself to a different interpretation, one that invokes a process of adaptive gain control. A singular feature of sensory systems is that their response to a stimulus changes across the course of exposure (Clifford and Rhodes, 2005). Contemporary descriptions of these changes view them as adaptive shifts in gain control that optimize the range of the sensitivity of the sensory system to the statistics of the environment (Wainwright, 1999). Because the shift in sensory gain sacrifices absolute sensitivity for differential sensitivity, a hallmark of adaptive gain control is the improvement in discrimination for stimuli in the range of the adapting stimulation accompanied by a decrement in detection or discrimination of near-threshold stimuli (Clifford, 2002) (Fig. 4). This was confirmed in experiments 3-5: presentation of an adapting suprathreshold vibration before each test stimulus elevated detection thresholds and near-threshold discrimination thresholds but lowered thresholds for discriminating between higher-amplitude vibrations. The important finding in the present experiments is that the very same pattern of changes was observed after noninformative vision of the hand (this view improved discrimination between high-amplitude vibrations but

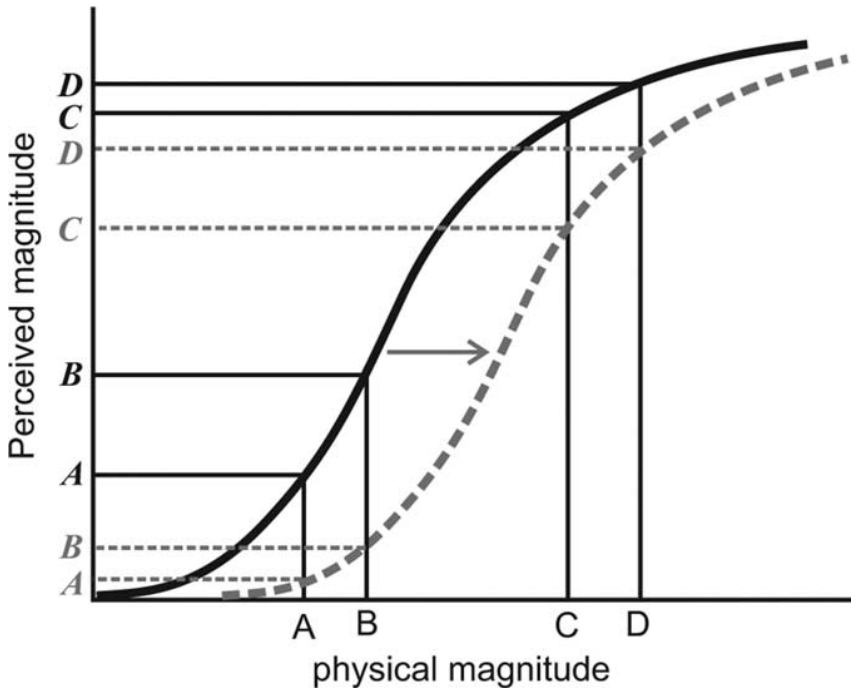

Figure 4. Schematic illustration of an adaptive rightward shift in the psychometric curve relating the physical magnitude of a stimulus to its perceived magnitude. Before adaptation (black solid line), the gain control is tuned to respond to all input magnitudes and thus presents a perception to a stimulus at point $A$ that is both detectable and can be discriminated from another stimulus at $B$, but this gain control presents poorly discriminable perceptions at $C$ and $D$. After adaptation, the gain is shifted (gray broken line) such that the perception at $A$ is now very weak (undetectable) and poorly discriminable from $B$, but the perceptions at $C$ and $D$ are more discriminable.

impaired detection and discrimination of near-threshold vibrations). Indeed, these effects of noninformative vision summated with the effects of the adapting vibration. Thus, noninformative vision produces changes in tactile sensitivity that resemble those observed after adaptation to tactile stimulation, and the two adaptive shifts combine additively to regulate tactile sensitivity.

How might visual information about a body part induce adaptive shifts in tactile sensitivity? We propose that the effects reported here reflect adaptive shifts in gain control operating within a bimodal system that receives both visual and somatosensory inputs. Electrophysiological recordings in the posterior parietal cortex of behaving monkeys have identified populations of neurons that respond to both visual and tactile stimuli with congruent receptive fields (Iriki et al., 1996; Duhamel et al., 1998; Obayashi et al., 2000; Avillac et al., 2005). For example, Iriki et al. (1996) described neurons in the anterior bank of the intraparietal sulcus that respond to tactile stimulation on the contralateral hand and also respond to visual stimuli in the vicinity of that hand, and the visual receptive fields of these neurons "follow" the hand as it moves. This bimodal system may contribute directly to tactile perception, or it may influence responses within somatosensory regions in the anterior parietal cortex (Taylor-Clarke et al., 2002; Ro et al., 2004; Fiorio and Haggard, 2005). In line with physiological evidence, these bimodal neurons would be activated by somatosensory input alone (e.g., during the adapting vibration while view of the hand was obscured by an opaque surface) and also by visual input alone (when the participant viewed their hand while no tactile stimuli were presented), and these inputs would combine when participants see their hand during tactile stimulation. It should be clear how this description explains the present results, because both the sight of the hand and the prolonged vibrotactile input would be independently capable of inducing adaptive shifts in the response properties of the bimodal neurons. These adaptive shifts would be manifest behaviorally as a loss in sensitivity for near-threshold stimuli but 
an increase in discriminability of suprathreshold stimuli. Moreover, if these adaptive shifts decrease absolute sensitivity by reducing gain in somatosensory cortical neurons (Fiorio and Haggard, 2005), this would reduce the receptive field size of those neurons, which could account for improvements in tactile spatial acuity (Taylor-Clarke et al., 2002; Haggard et al., 2003; Press et al., 2004; Serino et al., 2007).

In summary, the findings reported here identify a novel means by which vision of the body can alter tactile perception. We suggest that visual input can induce an adaptive shift in tactile sensitivity, evident as an impairment in detection and discrimination of near-threshold stimulation but a concurrent improvement in discrimination between suprathreshold stimuli. We believe this effect of vision on touch reflects the adaptive adjustment to gain control mechanisms operating with a bimodal (visuo-tactile) system.

\section{References}

Avillac M, Deneve S, Olivier E, Pouget A, Duhamel J-R (2005) Reference frames for representing visual and tactile locations in parietal cortex. Nat Neurosci 8:941-949.

Clifford CWG (2002) Perceptual adaptation: motion parallels orientation. Trends Cogn Sci 6:136-143.

Clifford CWG, Rhodes G, eds (2005) Fitting the mind to the world: aftereffects in high-level vision. Oxford: Oxford UP.

Duhamel J-R, Colby CL, Goldberg ME (1998) Ventral intraparietal area of the macaque: congruent visual and somatic response properties. J Neurophysiol 79:126-136.

Ernst MO, Banks MS (2002) Humans integrate visual and haptic information in a statistically optimal fashion. Nature 415:429-433.

Fiorio M, Haggard P (2005) Viewing the body prepares the brain for touch: effects of TMS over somatosensory cortex. Eur J Neurosci 22:773-777.

Gescheider GA, Bolanowski SJ, Hardick KR (2001) The frequency selectivity of information-processing channels in the tactile sensory system. Somatosens Motor Res 18:191-201.

Haggard P, Taylor-Clarke M, Kennett S (2003) Tactile perception, cortical representation and the bodily self. Curr Biol 13:R170-R173.

Iriki A, Tanaka M, Iwamura Y (1996) Coding of modified body schema during tool use by macaque postcentral neurones. NeuroReport 7:2325-2330
Kennett S, Taylor-Clarke M, Haggard P (2001) Noninformative vision improves the spatial resolution of touch in humans. Curr Biol 11:1188-1191.

Kontsevich LL, Tyler CW (1999) Bayesian adaptive estimation of psychometric slope and threshold. Vision Res 39:2729-2737.

LaMotte RH, Mountcastle VB (1975) Capacities of humans and monkeys to discriminate vibratory stimuli of different frequency and amplitude: a correlation between neural events and psychological measurements. J Neurophysiol 38:539-559.

Lederman SJ, Klatzky RL (2004) Multisensory texture perception. In: The handbook of multisensory processes (Calvert G, Spence C, Stein BE, eds), pp 107-122. Cambridge, MA: MIT.

Loftus GR, Masson MEJ (1994) Using confidence intervals in withinsubject designs. Psychon Bull Rev 1:476-490.

Mahns DA, Perkins NM, Sahai V, Robinson L, Rowe MJ (2006) Vibrotactile frequency discrimination in human hairy skin. J Neurophysiol 95:1442-1450.

Obayashi S, Tanaka M, Iriki A (2000) Subjective image of invisible hand coded by monkey intraparietal neurons. NeuroReport 11:3499-3505.

Press C, Taylor-Clarke M, Kennett S, Haggard P (2004) Visual enhancement of touch in spatial body representation. Exp Brain Res 154:238-245.

Ro T, Wallace R, Hagedorn J, Farne A, Pienkos E (2004) Visual enhancing of tactile perception in the posterior parietal cortex. J Cogn Neurosci 16:24-30.

Serino A, Farne A, Rinaldesi ML, Haggard P, Ladavas E (2007) Can vision of the body ameliorate impaired somatosensory function? Neuropsychologia 45:1101-1107.

Talbot WH, Darian-Smith I, Kornhuber HH, Mountcastle VB (1968) The sense of flutter-vibration: comparison of the human capacity with response patterns of mechanoreceptive afferents from the monkey hand J Neurophysiol 31:301-334.

Taylor-Clarke M, Kennett S, Haggard P (2002) Vision modulates somatosensory cortical processing. Curr Biol 12:233-236.

Taylor-Clarke M, Kennett S, Haggard P (2004a) Persistence of visual-tactile enhancement in humans. Neurosci Lett 354:22-25.

Taylor-Clarke M, Jacobsen P, Haggard P (2004b) Tactile perception, cortical representation and the bodily self. Nat Neurosci 19:219-220.

Tommerdahl M, Hester KD, Felix ER, Hollins M, Favorov OV, Quibrera PM, Whitsel BL (2005) Human vibrotactile frequency discriminative capacity after adaptation to $25 \mathrm{~Hz}$ or $200 \mathrm{~Hz}$ stimulation. Brain Res 1057:1-9.

Wainwright MJ (1999) Visual adaptation as optimal information transmission. Vision Res 39:3960-3974. 